\title{
Impact of External Phenomena In Compressed Sensing Methods For Wireless Sensor Networks
}

\author{
Michal Kochláñ \\ Department of Technical Cybernetics \\ Faculty of Management Science and Informatics \\ University of Žilina \\ Univerzitná 8215/1, 01026 Žilina, Slovakia \\ Email: michal.kochlan@fri.uniza.sk
}

\author{
Michal Hodoň \\ Department of Technical Cybernetics \\ Faculty of Management Science and Informatics \\ University of Žilina \\ Univerzitná 8215/1, 01026 Žilina, Slovakia \\ Email: michal.hodon@fri.uniza.sk
}

\begin{abstract}
Compressed sensing represents an interesting approach in signal processing and reconstruction. The theory involves a surprising number of branches of mathematics: linear algebra, functional analysis, convex and non-convex optimization, nonlinear approximation theory and probability. In general, the applications of compressed sensing can be found (or searched) wherever it is possible to express the signal in sparse representation in a "standard" base or in a base that was adjusted for particular signal. Core applications of compressed sensing today include image processing, signal denoising, image deblurring and inpainting. This paper addresses analysis the influence of external phenomena on the signal reconstruction using compressed sensing in wireless sensor networks. Such external phenomena include, for instance, additive white Gaussian noise (AWGN), attenuation or time shift. Three acoustic input signals sparse in frequency domain are used in experiments. The first one with significant frequency band from $500 \mathrm{~Hz}$ up to $700 \mathrm{~Hz}$. The second signal with one significant frequency band from $2400 \mathrm{~Hz}$ up to $3100 \mathrm{~Hz}$ with considerable frequency bands between $0 \mathrm{~Hz}$ to $1000 \mathrm{~Hz}$ and $5000 \mathrm{~Hz}$ to $6000 \mathrm{~Hz}$. The third signal used is a synthesized artificial sound invented for the experiment purposes only. It is strictly sparse in the frequency domain and has exactly three frequency bands between $400 \mathrm{~Hz}$ and $500 \mathrm{~Hz}$, $2000 \mathrm{~Hz}$ and $2100 \mathrm{~Hz}, 9000 \mathrm{~Hz}$ and $9100 \mathrm{~Hz}$. The results show that additive noise as well as attenuation have significant effect on the reconstruction accuracy using the selected distribution scenario and reconstruction method. On the other side, the time shift has no significant effect on the reconstruction.
\end{abstract}

\section{INTRODUCTION}

A WSN is a distributed system. Having this in mind, WSNs can be used for distributing of compressed sensing task [1]. This can be achieved such that the sensor nodes perform the sampling part of the compressed sensing. The sinking node(s) perform(s) the reconstruction part (see Fig. 1).

According to the basic definition of compressed sensing, it is a modern method for signal representation and data compression. It is based on the assumption that some (sparse) signals can be reconstructed from such series of samples that are considered to be incomplete [2], i.e. have insufficient information value for proper signal reconstruction through the sampling theorem. Such reconstruction is made of small amount of samples - less than the sampling theorem determines.

The cornerstone of signal reconstruction using compressed sensing - the $\ell_{1}$-minimization [2], which looks for the optimal

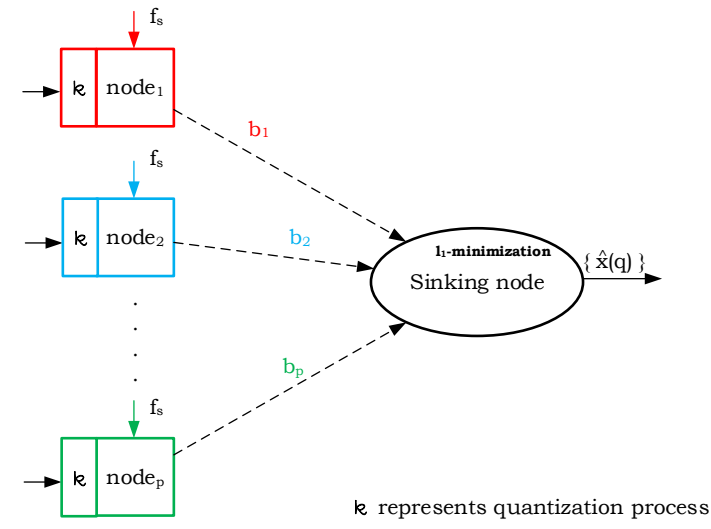

Fig. 1. Distributing compressed sensing task over the wireless sensor nodes (Compressed Sensing Method With Periodic Sampling Utilization)

representation of the signal in such base, where the signal is sparse. In compressed sensing, the measurement matrix $\boldsymbol{A}$ replaces the sampling process. This matrix determines the weight of each sample that enters the reconstruction process using $\ell_{1}$-minimization [3]. Sparse vector $\boldsymbol{x}$ represents samples of the sensed signal. The reduced vector $\boldsymbol{b}$ is a condensed version of $\boldsymbol{x}$. The vector $\boldsymbol{b}$ is a product of multiplication of the measurement matrix $\boldsymbol{A}$ and the vector of samples - $\boldsymbol{x}$ [3].

The wireless sensor networks are deployed in the real environment. In this environment, a sensed signal is being constantly influenced by different effects. In other words, the success of the signal reconstruction depends on the influence of external factors as well [4]. In case of WSNs, these factors include mainly noise, signal attenuation, wireless nodes' asynchronous operation as well as signal time-shift due to the spacial distribution of sensor nodes. Therefore, it is important to reveal the impact of these phenomena on the measure of accuracy of the original signal reconstruction. The term measure of accuracy of the original signal reconstruction is in this paper understood as a sum of squares from the difference of the original signal $x(i)$ and the reconstructed signal $\hat{x}(i)$. It is knows as mean squared error, and in this paper it is marked as $\mu$ : 

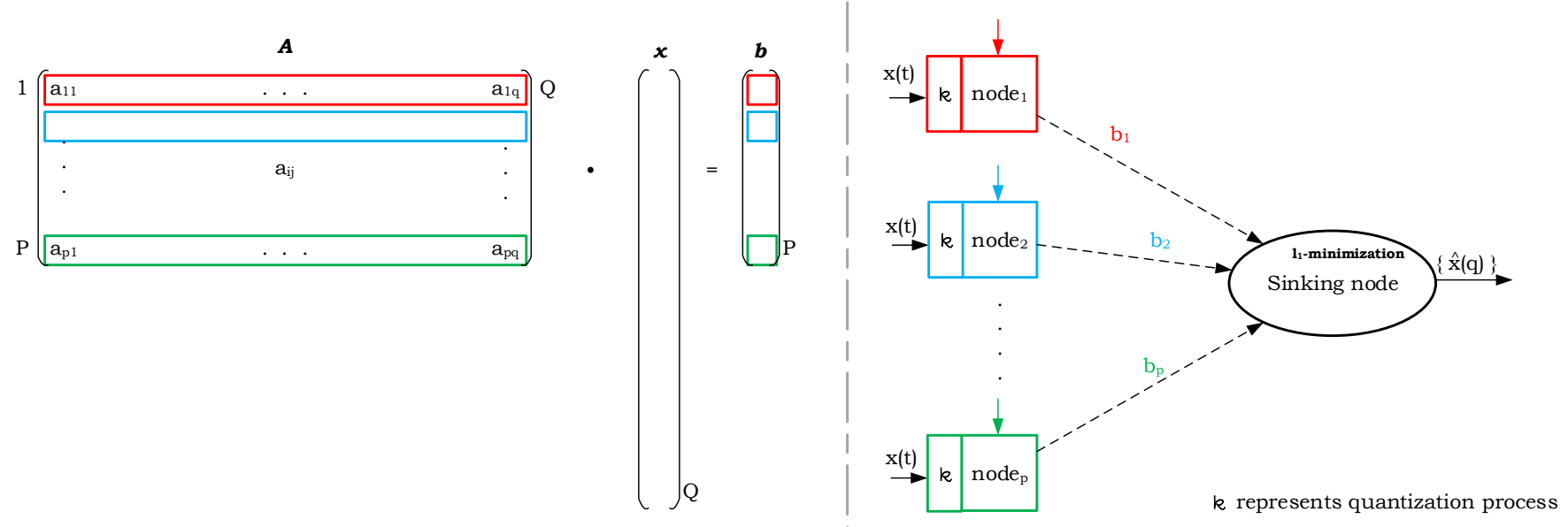

Fig. 2. Illustration of general idea of the Compressed Sensing Method With Periodic Sampling

$$
\frac{1}{N} \cdot \sum_{i=1}^{N}(x(i)-\hat{x}(i))^{2}=\mu .
$$

The the method for WSNs (described in the further text) is based on the fundamental concept of compressed sensing. This includes measurement matrix $\boldsymbol{A}$, vector of measured samples $\boldsymbol{x}$ and a reduced vector of samples $\boldsymbol{b}$. The actual reconstruction of the original signal is based on $\ell_{1}$-minimization. This minimization is being performed on the sink node(s).

\section{Compressed Sensing Method (With Periodic SAMPLING)}

General idea of the compressed sensing is illustrated on the figure 2. Let's assume that the signal is sampled at equidistant time instants, i.e. periodically. The result of sampling of the input signal $\boldsymbol{x}(t)$ is a sequence of samples $\{\boldsymbol{x}(q)\}$ with length $Q$. This sequence of samples is multiplied by the measurement matrix $\boldsymbol{A}$ which has the size $P \times Q$ where $P \ll Q$.

The product of this multiplication is a reduced vector of the samples $\boldsymbol{b}$ with length $P$. Such vector enters the reconstruction process using $\ell_{1}$-minimization. The resulting sequence $\{\hat{\boldsymbol{x}}(q)\}$ represents the reconstructed signal.

The elements of the measurement matrix $a_{i j}$ can be randomly generated [5] as:

- Elements with Gauss coefficients (independently generated from a normal distribution with zero mean and variance $\sigma_{\nu}$, i.e. $\left.\mathcal{N}\left(0, \sigma_{\nu}\right)\right)$;

- Bernoulli coefficients (elements that have values $\pm \frac{1}{\sqrt{\sigma_{\nu}}}$ );

- Binary values $\{0,1\}$ with matrix $\operatorname{sparsity}^{1} \mathcal{S}$.

It should be noted that the choice of the measurement matrix $\boldsymbol{A}$ is itself a very difficult problem. Literature suggests that the deterministic measurement matrix for specific applications is almost impossible to construct, therefore randomly generated matrices are used in practical scenarios [5], [6], [7]. The

\footnotetext{
${ }^{1}$ Matrix sparsity indicates the ratio of non-zero matrix elements over the zero ones.
}

optimal choice of the statistical properties of the measurement matrices is investigated by many researches with multiple approaches [6], [7], [8], [9], [10]. In case of the Gaussian matrix, the literature states that for matrix with dimensions $P \times Q$, the elements should be generated from a normal (Gaussian) distribution with zero mean and variance equal to either:

$$
\begin{aligned}
& \text { - } \sigma_{\nu}=\frac{1}{Q} \text {, i.e } \mathcal{N}\left(0, \frac{1}{Q}\right) \text { or, } \\
& \text { - } \sigma_{\nu}=\frac{1}{P} \text {, i.e } \mathcal{N}\left(0, \frac{1}{P}\right) \text { but also, } \\
& \text { - } \sigma_{\nu}=1 \text {, i.e } \mathcal{N}(0,1) \text {. }
\end{aligned}
$$

Now, let's suppose that each sensor node senses the same signal. General idea of the investigated method stands on the fact that each sensor node carries out multiplication of one line of the same measurement matrix with the measured samples [11]. The result is that a single node produces only a single coefficient that represents one element of the reduced vector $\boldsymbol{b}$ (see Fig. 2). This coefficient is then being sent to the sink node where the reconstruction takes place. At first, the sink node composes the reduced vector and then, this vector enters the process of reconstruction by $\ell_{1}$-minimization.

\section{CONDITIONS}

In order to investigate the behavior of the mentioned method, several experiments investigating performance under external phenomena have been carried out.

The following three input signals have been used, which all of them are sparse in frequency domain:

- Simplified version of the sound of Northern Raven;

- Simplified version of the sound of Bohemian Waxwing;

- An artificial signal designed especially as sparse in the frequency domain. This signal is marked as Artificial sparse signal.

The first signal is one with a single significant frequency band starting at around $500 \mathrm{~Hz}$ and ending at around $700 \mathrm{~Hz}$. This signal is simplified and short version of the sound of 
the Northern Raven ${ }^{2}$ - (Corvus corax). The Discrete Fourier Transform (DFT) is shown on Fig. 3.



Fig. 3. Simplified sound of the Northern Raven - a sparse signal in frequency domain shown in the frequency domain (a single significant frequency band, $500 \mathrm{~Hz}-700 \mathrm{~Hz}$

The second signal that is used in the simulations as an input signal $x(t)$ is a simplified and shortened version of the sound of Bohemian Waxwing ${ }^{3}$ - (Bombycilla garrulus). The used signal has one significant frequency band starting at around $2400 \mathrm{~Hz}$ and ending at around $3100 \mathrm{~Hz}$. However, there are also frequency bands that should be considered such as the ones in $0 \mathrm{~Hz}-1000 \mathrm{~Hz}$ and $5000 \mathrm{~Hz}-6000 \mathrm{~Hz}$. The DFT of the signal is shown on Fig. 4.

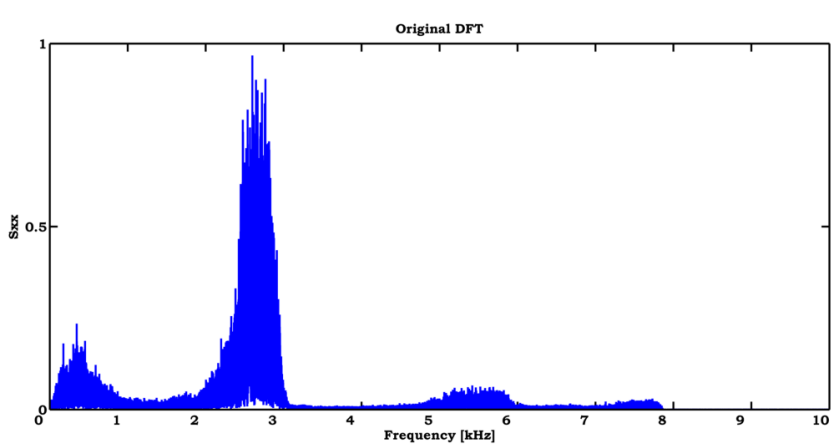

Fig. 4. Simplified sound of the Bohemian Waxwing - a sparse signal in frequency domain shown in the frequency domain (significant frequency band $2400 \mathrm{~Hz}-3100 \mathrm{~Hz})$

The third signal used as an input for the simulations is a synthesized artificial sound invented just for the experiment purposes only. It is strictly sparse in the frequency domain. It has three frequency bands: $400 \mathrm{~Hz}-500 \mathrm{~Hz}, 2000 \mathrm{~Hz}-$ $2100 \mathrm{~Hz}$ and $9000 \mathrm{~Hz}-9100 \mathrm{~Hz}$. This signal was invented for the reference and comparison of the performance of the compressed sensing with real-world sparse signals. The DFT of the signal is shown on Fig. 5.

All three presented signals are shown in the $10 \mathrm{kHz}$ bandwidth. This means that a sampling scheme that can be used without significant signal reconstruction error is to sample

\footnotetext{
${ }^{2}$ The original sound of the Northern Raven is from Xeno-Canto sound database. http://www.xeno-canto.org/124411

${ }^{3}$ The original sound of the Bohemian Waxwing is from Xeno-Canto sound database. http://www.xeno-canto.org/121467
}



Fig. 5. Artificial sparse signal - a sparse signal in frequency domain shown in the frequency domain (significant frequency bands $400 \mathrm{~Hz}-500 \mathrm{~Hz}, 2000 \mathrm{~Hz}$ $-2100 \mathrm{~Hz}$ and $9000 \mathrm{~Hz}-9100 \mathrm{~Hz})$

with sampling frequency equal to $20 \mathrm{kHz}$ (this comes out from the sampling theorem). This produces 20000 samples per second (sps). The worst case scenario is to transmit all the samples to the sinking node so that the reconstruction can be performed there. However, the highest frequency component of the Northern Raven signal is $2150 \mathrm{~Hz}$. In the case of Bohemian Waxwing, the highest frequency component of this particular signal is $7950 \mathrm{~Hz}$. The highest frequency component of the artificial sparse signal is $9100 \mathrm{~Hz}$. Knowing the basic information about the sampled signals, it can be concluded that the first signal - simplified version of "Northern Raven" signal - can be sampled as low as $4.3 \mathrm{kHz}$ which results in producing 4300 samples per second (sps) only. Similarly, the second signal - simplified version of "Bohemian Waxwing" signal - can be sampled by sampling frequency $15.9 \mathrm{kHz}$, which produces 15900 samples per second (sps). The third signal, an artificial sparse signal in frequency domain that has been introduced for the simulation purposes only, has to be sampled by at least $18.2 \mathrm{kHz}$ sampling frequency.

Experiment of compressed sensing that utilizes periodic sampling is based on a network consisting of $P$ sensor nodes. These nodes perform sampling at periodic intervals, i.e. with sampling frequency $f_{s}$. This sampling frequency is the same on all nodes. The sampling process generates a discrete sequence $\{x(q)\}$ that has $Q$ elements. This sequence $\{x(q)\}$ is multiplied by the measurement matrix. In this experiment, the measurement matrix is based on random Gaussian values. The measurement matrix $\boldsymbol{A}_{\boldsymbol{P} \times \boldsymbol{Q}}$ is of size $P \times Q$ and its elements have values from the normal (Gaussian) distribution $\mathcal{N}\left(0, \frac{1}{P}\right)$.

Let's consider the situation where the input signal is the same for all sensor nodes. Compressed sensing task can be parallelized such as each node performs sensing such as each sensor executes operation corresponding to a single row of the measurement matrix $\boldsymbol{A}_{\boldsymbol{P} \times \boldsymbol{Q}}$. There are $P$ nodes in the sensor network. Each node ( $i-t h$ node) performs a series of $Q$ measurements:

$$
\sum_{j=1}^{Q} a_{i j} \cdot x_{j}=b_{i} ; i \in\langle 1 ; P\rangle .
$$


The above relation reduces the sequence of measurements into a single coefficient. The relation produces $i-t h$ coefficient of the reduced vector $\boldsymbol{b}$, i.e. $b_{i}$. These coefficients $\left\{b_{1}, \ldots, b_{i}, \ldots b_{p}\right\}$ from all nodes in the WSN are being sent to the sink node and in the sink node, reconstruction of the vector $\boldsymbol{b}$ takes place. Afterwards, the reconstruction of the original sensed signal is performed. This reconstruction is based on the $\ell_{1}$-minimization [12]. The reconstruction based on the $\ell_{1}$ minimization produces a discrete sequence representing the original sensed signal $\{\hat{x}(n)\} n=1,2, \ldots, Q$.

In this particular case, the measurement matrix $\boldsymbol{A}$ has $1000 \times 20000=20 \mathrm{mil}$. elements, i.e. $P=1000$ and $Q=20000$. Thus, this simulates a thousand nodes performing compressed sensing in the network. The elements of the measurement matrix are randomly generated Gaussian values from the normal distribution with zero mean value and variance $\frac{1}{1000}$, i.e. $\mathcal{N}\left(0, \frac{1}{1000}\right)$. Dimension $Q$ of the matrix is chosen with respect to the sampling theorem.

For the experiment purposes, the measurement matrix is generated as a single entity, i.e. not partially at each node. Each sensor node performs the operations that correspond to the matrix row for the particular node. Using this row, each node performs calculation of the coefficient $b_{i}$ and transmits this coefficient to the sink node.

\section{ACHIEVED Results}

For the experiment purposes and for investigation of the behavior on the influence of the external phenomena, the following phenomena are being investigated:

- Additive White Gaussian Noise;

- attenuation;

- time shift of the input signal;

In the simulation on the influence of the AWGN, the white noise with zero mean value and normal distribution designated as $W N_{0}$ is considered. At the input of the individual sensors, there is input signal $x_{i}(t)$ with mutually uncorrelated noise $e_{i}(t)$ as follows:

$$
x_{i}(t)=x(t)+e_{i}(t) .
$$

The conducted experiment investigates the influence of the noise on the measure of accuracy of the original signal reconstruction $\mu$. In particular, it shows the dependency of $\mu$ on the statistical distribution of the AWGN, see Fig. 6. The statistical distribution of the additive white noise has a zero mean value and variance of normal (uniform) distribution $\sigma_{i}=\frac{T_{s}}{i} ; i \in\langle 2,3,4, \ldots, 10\rangle$. Parameter $T_{s}$ represents the period of the sampling frequency $f_{s}$. Since the sampling frequency $f_{s}=20 \mathrm{kHz}$, the sampling period $T_{s}=50 \mu \mathrm{s}$.

The numerical results show that as the variance of the normal distribution of the AWGN grows, the measure of the accuracy of the reconstruction increases as well. Significant change of the mean squared error of the signal reconstruction is located between $\sigma_{i}$ values $\frac{T_{s}}{8}$ and $\frac{T_{s}}{7}$. The overall performance of this method in signal reconstruction degrades from $6.94 \%$ error rate at $\sigma_{i}=\frac{T_{s}}{8}$ to $14.56 \%$ error rate at $\sigma_{i}=\frac{T_{s}}{7}$.

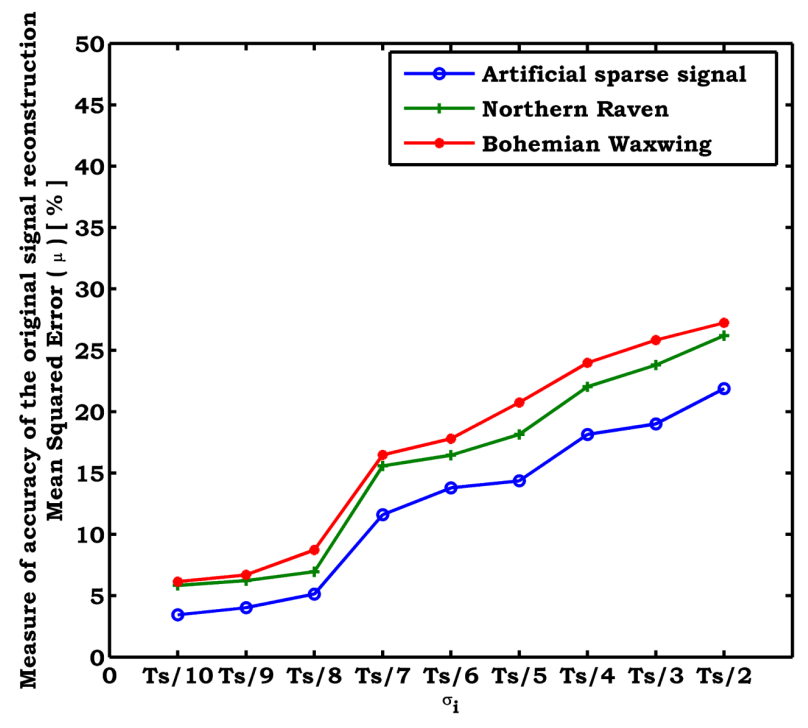

Fig. 6. Dependency of the measure of accuracy of the original signal reconstruction $\mu$ on the statistical distribution of the AWGN represented by white noise variance $\sigma_{i}$

TABLE I

NUMERICAL RESULTS OF $\mu$ [\%] DEPENDING ON THE STATISTICAL DISTRIBUTION OF THE AWGN REPRESENTED BY WHITE NOISE VARIANCE $\sigma_{i}$

\begin{tabular}{lccccccccc}
\hline Input signal $\backslash \sigma_{i}$ & $\frac{T_{s}}{10}$ & $\frac{T_{s}}{9}$ & $\frac{T_{s}}{8}$ & $\frac{T_{s}}{7}$ & $\frac{T_{s}}{6}$ & $\frac{T_{s}}{5}$ & $\frac{T_{s}}{4}$ & $\frac{T_{s}}{3}$ & $\frac{T_{s}}{2}$ \\
\hline Artificial sparse signal & 3.44 & 4.03 & 5.13 & 11.60 & 13.80 & 14.37 & 18.15 & 18.99 & 21.87 \\
\hline Northern Raven & 5.83 & 6.24 & 6.96 & 15.59 & 16.45 & 18.15 & 22.04 & 23.79 & 26.20 \\
\hline Bohemian Waxwing & 6.15 & 6.70 & 8.73 & 16.48 & 17.80 & 20.74 & 23.98 & 25.83 & 27.23 \\
\hline
\end{tabular}

For AWGN variance up to around $\frac{T_{s}}{8}$ the mean squared error of the reconstruction keeps under $10 \%$, for Artificial sparse signal the average reconstruction error equals $4.20 \%$. However, this is the synthetic signal. On the other hand, reallife signals perform a little worse, e.g. simplified sound of the Northern Raven has average value of mean squared error of the reconstruction $6.34 \%$ for in the AWGN variance up to around $\frac{T_{s}}{8}$. Simulation of reconstruction of the simplified sound of the Bohemian Waxwing has the average equal to $7.19 \%$ in the same variance limit.

The next conducted experiment investigates the dependency of $\mu$ on the Signal-to-Noise Ratio (SNR), see Fig. 7. The noise level begins at $30 \mathrm{~dB}$ and degrades to $10 \mathrm{~dB}$. AWGN with zero mean value and variance $\sigma=\frac{T_{s}}{8}$ has been used in this simulations. Parameter $T_{s}$ denotes period of the sampling frequency $f_{s}$, which equals $20 k H z$.

TABLE II

NUMERICAL RESULTS OF THE MEASURE OF ACCURACY OF THE ORIGINAL SIGNAL RECONSTRUCTION $\mu$ [\%] DEPENDING ON THE SNR

\begin{tabular}{lccccccccccc}
\hline Input signal $\backslash S N R[d B]$ & $\mathbf{1 0}$ & $\mathbf{1 2}$ & $\mathbf{1 4}$ & $\mathbf{1 6}$ & $\mathbf{1 8}$ & $\mathbf{2 0}$ & $\mathbf{2 2}$ & $\mathbf{2 4}$ & $\mathbf{2 6}$ & $\mathbf{2 8}$ & $\mathbf{3 0}$ \\
\hline Artificial sparse signal & 34.64 & 30.10 & 25.14 & 18.16 & 14.43 & 10.46 & 7.26 & 6.69 & 6.11 & 4.64 & 3.96 \\
\hline Northern Raven & 36.53 & 32.24 & 29.37 & 22.21 & 16.76 & 13.46 & 8.69 & 7.48 & 7.59 & 5.77 & 4.55 \\
\hline Bohemian Waxwing & 37.76 & 35.51 & 32.96 & 23.88 & 17.86 & 14.59 & 9.08 & 8.50 & 7.82 & 6.42 & 5.43 \\
\hline
\end{tabular}

The results reveal that the measure of the accuracy of 


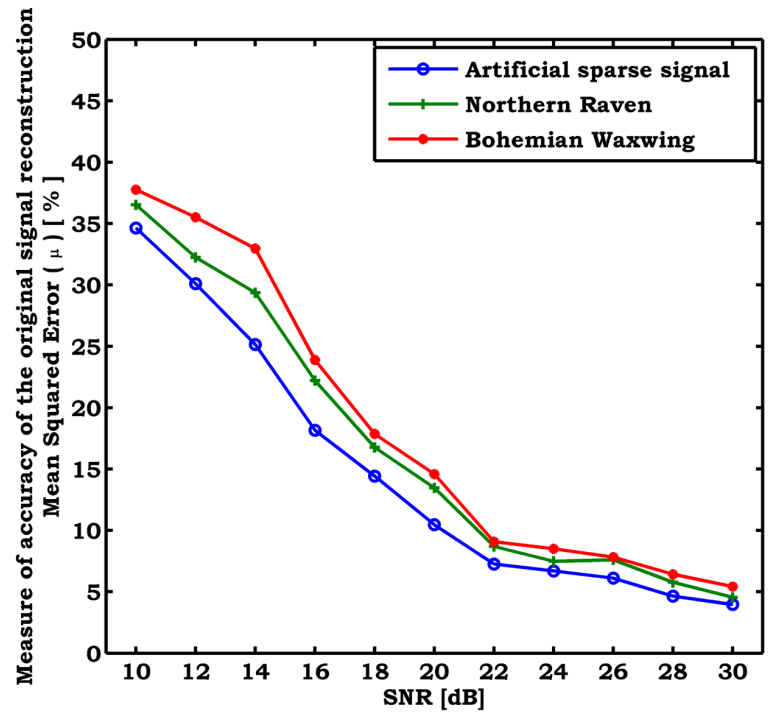

Fig. 7. Dependency of the measure of accuracy of the original signal reconstruction $\mu$ on the SNR

the reconstruction $(\mu)$ via mentioned reconstruction method performs good when SNR keeps above $22 d B$. Then, the mean squared error of the reconstruction stays below $10 \%$. For example, the average error for Artificial sparse signal is $5.73 \%$ when SNR stays above $22 d B$. Simplified sound of the Northern Raven has average value of mean squared error in the same range $6.82 \%$. Similarly, simplified sound of the Bohemian Waxwing has the average error $7.45 \%$ for SNR more than $22 d B$. The overall performance on reconstruction error of the proposed method on all investigated input signals is $6.67 \%$ for SNR more than $22 d B$.

This experiment as well as the previous one proves that the described reconstruction method does not suit well for reconstruction of acoustic signals in noisy environment. Advanced processing techniques for noise suppression has to be utilized. Having low noise acoustic input signals, this method provides good reconstruction performance with error up to $10 \%$. Having environment with SNR higher than $22 d B$ enables this method to perform with good results. Then, the mean squared error of the original signal reconstruction stays below $10 \%$.

Another phenomenon that influences the sensed signal is called attenuation. In other words, the attenuation modifies the amplitude(s) of the input signal - it scales the original signal on the inputs of the nodes. This phenomenon can be expressed mathematically as the following relation:

$$
x_{i}(t)=k_{i} \cdot x(t) .
$$

In this experimental scenario each of the sensors in the network senses an input signal which is scaled by the scaling coefficients $k_{i}$. These coefficient are randomly generated from normal (Gaussian) statistical distribution with mean value equal to 0.5 and with variance equal to 1 , i.e. $\mathcal{N}(0.5,1)$. Based on their values, several groups with different root mean square
(RMS) values have been formed. The output of this simulation is the dependency of the measure of accuracy of the original signal reconstruction $\mu$ on the effective value (RMS) of the scaling coefficients $k_{r m s}$, as shown on Fig. 8.

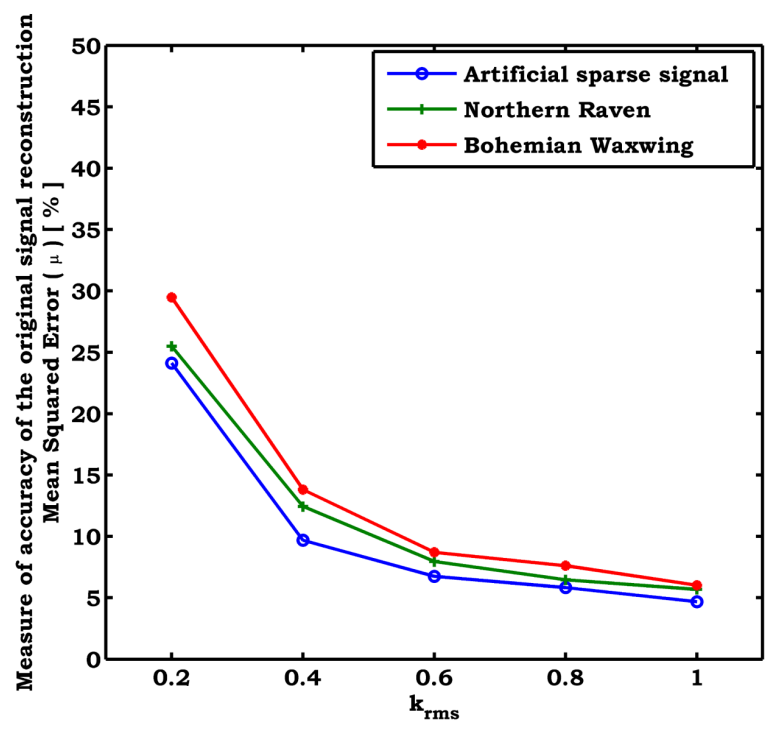

Fig. 8. Dependency of the measure of accuracy of the original signal reconstruction $\mu$ on effective value of the scaling coefficients $k_{r m s}$ (performed with Compressed Sensing Method Using Periodic Sampling)

TABLE III

NUMERICAL RESULTS OF THE MEASURE OF ACCURACY OF THE ORIGINAL SIGNAL RECONSTRUCTION $\mu$ REGARDING THE EFFECTIVE VALUE OF THE SCALING COEFFICIENTS $k_{r m s}$

\begin{tabular}{lccccc}
\hline Input signal $\backslash k_{r m s}$ & $\mathbf{0 . 2}$ & $\mathbf{0 . 4}$ & $\mathbf{0 . 6}$ & $\mathbf{0 . 8}$ & $\mathbf{1}$ \\
\hline Artificial sparse signal & 24.12 & 9.68 & 6.75 & 5.83 & 4.68 \\
\hline Northern Raven & 25.49 & 12.44 & 7.96 & 6.46 & 5.68 \\
\hline Bohemian Waxwing & 29.47 & 13.81 & 8.71 & 7.61 & 6.02 \\
\hline
\end{tabular}

The results show significant increase of the reconstruction error $\mu$ for the effective value of the scaling coefficients higher than 0.6. Significant change of the mean squared error of the signal reconstruction is located between $k_{r m s}$ values 0.6 and 0.4 . The overall performance of this method in signal reconstruction degrades from $7.81 \%$ error rate at $k_{r m s}=0.6$ to $11.98 \%$ error rate at $k_{r m s}=0.4$.

Having Artificial sparse signal, the average reconstruction error is $5.75 \%$ for effective value of the scaling coefficients from 0.6 to 1.0. The average reconstruction error within the same interval equals $6.70 \%$ for simplified sound of the Northern Raven. Simplified sound of the Bohemian Waxwing has the average error of the reconstruction in the range $0.6-1.0$ equal to $7.45 \%$.

It can be concluded that the mentioned reconstruction method performs well with attenuated signals that are attenuated not less than $60 \%$ of the amplitude in average.

Major impact on the success rate of the reconstruction in the sinking node has also signal shift at individual sensor nodes. This can be expressed as: 


$$
x_{i}(t)=x\left(t-\tau_{i}\right) .
$$

The shift $\tau_{i}$ expresses different distances of the nodes from a common source of the sensed signal. The coefficients of the time shift are for the experimental purposes generated from normal statistical distribution with a mean value equal to 0 and variance $\sigma_{i}=i \cdot T_{s} ; i \in\langle 1,2,3, \ldots, 10\rangle$. Parameter $T_{s}$ represents the period of the sampling frequency $f_{s}$, which equals $20 \mathrm{kHz}$. Thus, the sampling period $T_{s}=50 \mu \mathrm{s}$. The output of this experiment is the dependency of $\mu$ on the time shift expressed by $\sigma_{i}$, see Fig. 9 .

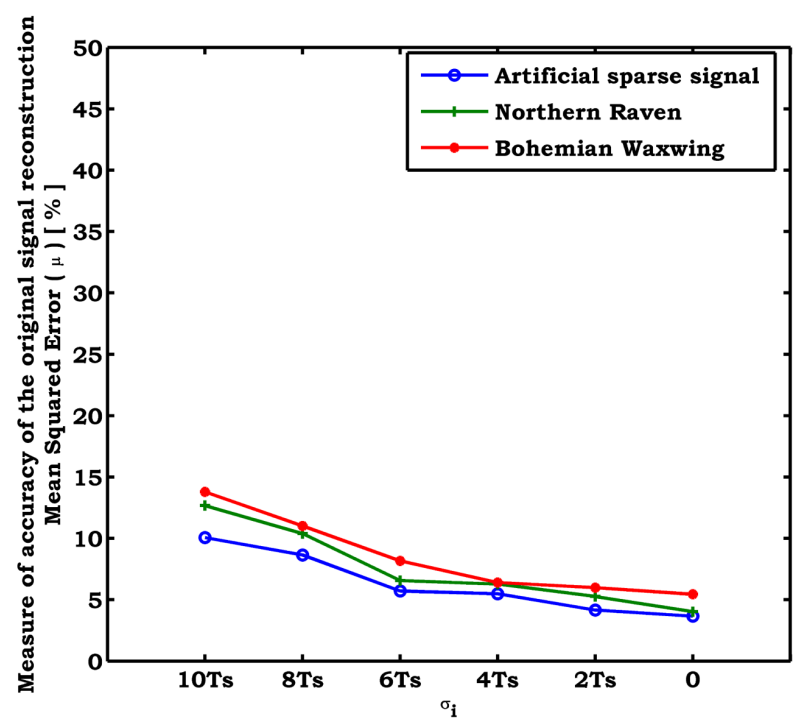

Fig. 9. Dependency of the measure of accuracy of the original signal reconstruction $\mu$ on the time shift variance $\sigma_{i}$

The results show that the accuracy of the original signal reconstruction $(\mu)$ performs very well for time shift up to 8 fold of the sampling period $T_{s}$.

The average error for Artificial sparse signal in all performed simulations is $6.29 \%$. Simplified sound of the Northern Raven has average value of mean squared error for all simulations of the time shift equal to $7.53 \%$. Simplified sound of the Bohemian Waxwing has the average error $8.47 \%$. The overall performance on reconstruction error of the proposed method on all investigated input signals is $5.60 \%$ for time shift variance $\sigma_{i}<8 \cdot T_{s}$.

Since the time shift of the input signal can vary in time, the results show mild resistance of the proposed method on the time shift of the input signal.

\section{Discussion on Achieved Results}

This method, Compressed Sensing Method Using Periodic Sampling, requires sampling on the periodic basis on all nodes in the WSN. Therefore, sampling performed on each of the nodes was done under sampling frequency $f_{s}=20 k \mathrm{~Hz}$. This produces 20000 samples per second. However, the reduction part of the proposed method (equation 2), reduces these 20
TABLE IV

NUMERICAL RESULTS OF THE MEASURE OF ACCURACY OF THE ORIGINAL SIGNAL RECONSTRUCTION $\mu$ [\%] DEPENDING ON THE TIME SHIFT VARIANCE $\sigma_{i}$

\begin{tabular}{lcccccc}
\hline Input signal $\backslash \sigma_{i}$ & $10 \cdot T_{s}$ & $8 \cdot T_{s}$ & $6 \cdot T_{s}$ & $4 \cdot T_{s}$ & $2 \cdot T_{s}$ & $0 \cdot T_{s}$ \\
\hline Artificial sparse signal & 10.06 & 8.65 & 5.71 & 5.49 & 4.16 & 3.66 \\
\hline Northern Raven & 12.67 & 10.39 & 6.56 & 6.28 & 5.26 & 4.03 \\
\hline Bohemian Waxwing & 13.79 & 11.02 & 8.17 & 6.40 & 5.99 & 5.45 \\
\hline
\end{tabular}

000 samples into a single one on each of the nodes. Having 1000 nodes in all experiments, the overall compression ratio is $1: 20$.

The investigated method requires only 1000 samples to be transmitted to the sink node in order to properly reconstruct the original signal with certain accuracy (in average $\mu<10 \%$ ). Therefore, from the point of reduction of the samples, necessary to be sent over the network for reconstruction in the sink node, the proposed method is 20 times better than the case where all the samples obtained using the sampling theorem. This can be applied for all investigated input signals.

The aforementioned applies for the sampling frequency $f_{s}=20 \mathrm{kHz}$. However, based on the nature of the figure investigated signals and their maximal frequency component, the input signals can be sampled by lower frequency using sampling theorem:

- simplified sound of the Northern Raven $f_{s_{-} \text {raven }}=$ $4.3 \mathrm{kHz}$;

- simplified sound of the Bohemian Waxwing $f_{s_{-} w a x w i n g}$ $=15.9 \mathrm{kHz}$

- Artificial sparse signal $f_{s_{-} \text {artificial }}=18.2 \mathrm{kHz}$.

Following the consideration that for the simplified sound of the Northern Raven only $f_{\text {s_raven }}$ is enough, then this proposed method is just 4 times better from the point of reduction of the samples necessary to be sent to the sink node. Similarly, considering $f_{s_{-} \text {waxwing }}$ and simplified sound of the Bohemian Waxwing as an input signal, this method gives almost 16 times better performance. Having Artificial sparse signal as an input signal and considering $f_{s_{-} \text {artificial }}$, this method is 18 times better regarding the reduction of the samples required to be sent to the sink node.

From the point of the sampling process, this method brings no saving of the sampled values over the sampling theorem. This comes out of form the method design since it uses sampling pattern based on the periodic sampling following the sampling theorem. This method saves only data that are being sent from the nodes to the sinking node.

\section{CONCLUSION}

The results show that the mentioned method for reconstruction of the acoustic signals does not suit well for the reconstruction of signals in noisy environment. For all three simulation scenarios and all three input signals, the dependency of the measure of the accuracy of the reconstruction $\mu$ has increasing and nonlinear character. This is obvious where mean squared error of the reconstruction is less than $10 \%$ 
only for SNR higher than $22 \mathrm{~dB}$. The variance of AWGN should be lower than $12.5 \%$ of the sampling period $T_{s}$. The experiments investigating effect of the AWGN and SNR show that the selected reconstruction method is sensitive to the noise. Therefore, it is more suitable for reconstructing signals in the environment with low noise levels.

The attenuation has significant effect only in case of higher attenuation level across the network. In other words, the described method can successfully reconstruct the original signal when the amplitude at most of the sensors is attenuated less than the $50 \%$ of the original signal's amplitude.

The simulations proved that time shift of the input signal does not significantly influence the reconstruction via compressed sensing methods. The mean squared error is mostly less than $10 \%$ for time shift up to ten fold of the sampling period. The time shift of the input signal can vary in time, thus, the results show mild resistance of the proposed methods on the time shift of the sensed signal.

Motivation of this work is related to the investigation of reconstruction methods form target localization WSN and distributed compressed sensing with perspective energy efficiency. The results of this paper show that Compressed Sensing Method using Periodic Sampling as described earlier can save the number of samples being sent to the sink node and thus reducing energy consumed by transmission. However, saving on signal processing does not come to effect since compressed sensing with periodic sampling requires periodic processing of the sensed values. Nevertheless, there are more compressed sensing methods for investigation, therefore, the future work includes utilization and performance comparison of Compressed Sensing Method Using Random Sampling
Generated By Measurement Matrix or Modified Compressed Sensing Method Using Random Sampling [13], [8].

\section{REFERENCES}

[1] I.F. Akyildiz and W. Su and Y. Sankarasubramaniam and E. Cayirc, "Wireless sensor networks: a survey", in Computer Networks, 2002 ISSN: 1389-1286, DOI: 10.1016/S1389-1286(01)00302-4

[2] M. A. Razzaque and Ch. Bleakley and S. Dobson, "Compression in wireless sensor networks: A survey and comparative evaluation", in ACM Transactions on Sensor Networks (TOSN), 2013, DOI: 10.1145/2528948

[3] C. Caione and D. Brunelli and L. Benini, "Distributed compressive sampling for lifetime optimization in dense wireless sensor networks", in Industrial Informatics, IEEE Transactions on, 2012, DOI: 10.1109/TII.2011.2173500

[4] P. Rawat and K. D. Singh and H. Chaouchi and J. M. Bonnin, "Wireless sensor networks: a survey on recent developments and potential synergies", in The Journal of supercomputing, 2014, DOI: 10.1007/s11227013-1021-9

[5] K. Hayashi and M. Nagahara and T. Tanaka, "A user's guide to compressed sensing for communications systems", in IEICE transactions on communications, 2013, DOI: 10.1587/transcom.E96.B.685

[6] S. Foucart and H. Rauhut, "An Invitation to Compressive Sensing", ISBN 978-0-8176-4948-7, 2013

[7] Y. C. Eldar and G. Kutyniok, "Compressed sensing: theory and applications", ISBN: 978-1107005587, 2012

[8] M. Fornasier and H. Rauhut, "Handbook of mathematical methods in imaging, Compressive sensing", p. 187 - 228, 2011, ISBN 978-0-38792920-0

[9] M. Fornasier and H. Rauhut, "Compressive sensing", 2011, ISBN: 9780387929200

[10] M. Elad, "Sparse and redundant representations: from theory to applications in signal and image processing", 2010, ISBN: 9781441970107

[11] A. C. Fannjiang and T. Strohmer and P. Yan, "Compressed remote sensing of sparse objects",2010, https://www.math.ucdavis.edu/ strohmer/papers/2009/CS-par.pdf

[12] E. J. Candes and M. B. Wakin, "An Introduction to Compressive Sampling" ISSN: 1053-5888, 2008, DOI: 10.1109/MSP.2007.914731

[13] A. Cohen and W. Dahmen and R. DeVore, "Compressed Sensing and Best k-term Approximation", in American Mathematical Society, Joirnal of the, Vol. 22, No. 1, p. 211-231, 2009, DOI: 10.1090/S0894-0347-0800610-3 